\title{
Microenseñanza en el laboratorio de ciencias para el alumnado del grado de educación infantil
}

\author{
Micro-teaching in the science laboratory for degree's students early childhood \\ education
}

Microensino no laboratório de ciências para alunos do ensino infantil

Carles Dulsat-Ortiz ${ }^{10}$

Recibido: abril de 2019

Aceptado: junio de 2019

Para citar este artículo: Dulsat-Ortiz, C. (2019). Microenseñanza en el laboratorio de ciencias para el alumnado del grado de educación infantil. Revista Científica, 36(3), 367-380. Doi: https://doi.org/10.14483/23448350.14769

\section{Resumen}

Esta investigación pretende acercarse al aprendizaje desde los pilares de Delors en un espacio tan específico como el laboratorio de ciencias, así como a la aplicación del concepto de la transposición didáctica de Chevallard. Para ello, se acerca a la utilización de la metodología activa: microenseñanza. El planteamiento de esta microdocencia se orienta a introducir los conocimientos científicos y mostrarlos mediante experimentos al resto del grupo clase. Por ello, la finalidad de esta investigación radicó en describir desde el punto de vista del alumnado las vivencias de esta metodología las ventajas que supone esta al ser más participativa. La manera como se hizo la recolección y posterior análisis de la información siguió una metodología no experimental solo pos-pos-prueba con grupo no equivalente y único, siendo la población invitada el alumnado de segundo A del grado de educación infantil en la Universidade da Coruña. Entre los resultados, destacamos el elevado grado de aceptación de la metodología y cómo esta ha conseguido aumentar el grado de aceptación inicial hacia las ciencias naturales. Entre las conclusiones, cabe destacar que la metodología resultó acertada en su aplicación porque así lo mostraron las alumnas en sus respuestas, así como también por el aumento en el acercamiento a las ciencias y adquisición de los distintos saberes.

Palabras clave: estudios universitarios, experimentos, innovación pedagógica, método activo.

\begin{abstract}
This research aims to approach learning from the pillars of Delors in a space as specific as the science laboratory as well as the application of the concept of Chevallard's didactic transposition. For this, it approaches the use of the active methodology: micro-education. The approach of this microteaching is oriented to introduce scientific knowledge and show them through experiments to the rest of the class group. Therefore, the purpose of this research is to describe from the point of view of students the experiences of this methodology and
\end{abstract}

Universidade da Coruña, España. carles.dulsat.ortiz@udc.es 
the advantages of this more participatory methodology. The method used for the collection and subsequent analysis of the information followed a non-experimental only post-test methodology with a non-equivalent and unique group, being the invited population the students of second $A$ of the degree of infantile education in the Coruña University. Among the results, we highlight the high degree of acceptance of the methodology and how it has managed to increase the degree of initial acceptance towards the natural sciences. Among the conclusions, it should be noted that the methodology was successful in its application because students showed it in their answers, also because of the increase in the approach to science and acquisition of different knowledge.

Keywords: activity method, educational innovation, experiment undergraduate, study.

\section{Resumo}

Esta pesquisa visa abordar a aprendizagem a partir dos pilares de Delors em um espaço tão específico quanto o laboratório de ciências, bem como a aplicação do conceito de transposição didática de Chevallard. Para isso, aborda o uso da metodologia ativa: micro-educação. A abordagem desta microdocência é orientada para introduzir o conhecimento científico e mostrá-los através de experimentos para o resto do grupo de classes. Portanto, o objetivo desta pesquisa é descrever, do ponto de vista dos alunos, as experiências dessa metodologia e as vantagens dessa metodologia máis participativa. O método utilizado para a coleta e posterior análise das informações seguiu uma metodologia non experimental somente pós-teste com um grupo não equivalente e único, sendo a população convidada os estudantes do segundo A do grau de educação infantil na Universidade de Coruña. Entre os resultados, destacamos o alto grau de aceitação da metodologia e como ela conseguiu aumentar o grau de aceitação inicial em relação às ciências naturais. Entre as conclusões, destaca-se que a metodologia foi bem sucedida na sua aplicação, pois os alunos a mostraram em suas respostas, inclusive em função do aumento da abordagem da ciência e da aquisição de diferentes conhecimentos.

Palavras-chaves: inovação pedagógica, superior primeiro grau, método activo, experiência.

\section{Introducción}

Esta investigación nació de la inquietud de aproximar la enseñanza de las ciencias de la naturaleza al alumnado del grado de educación infantil. La experiencia docente realizada con anterioridad, mediante metodología magistral en el laboratorio, se percibía como monótona, que no conseguía acercar el mundo y los conceptos de ciencias al alumnado. Se indagó en metodologías más participativas que dejaran atrás las sesiones de laboratorio repetitivas en las cuales se seguían unas pautas marcadas por parte del docente. Por lo tanto, se plantea un problema abierto que se inicia desde la práctica y la reflexión docente. Con esto, se busca generar un conocimiento del uso de metodologías más participativas para el alumnado.

Así pues, desde la experiencia docente, crece la inquietud e interés por la mejora del aprendizaje del alumnado, cambiando la metodología de la propia práctica en el laboratorio. De esta manera, se pretende aproximar el acto educativo hacia una fuente de creciente interés hacia los contenidos teóricos mediante la búsqueda de información, la manipulación y la experimentación. Todo esto parte del interés personal del alumnado hacia conceptos y ámbitos específicos de las ciencias de la naturaleza.

El problema planteado se inicia en una realidad percibida en las sesiones prácticas con el alumnado del grado de educación infantil en la materia de Enseñanza de las Ciencias de la naturaleza. Esta percepción requiere de un cambio en la metodología usada para su enseñanza. De ahí la realización de un estudio dirigido a solucionar las reticencias y resistencias iniciales del alumnado por las ciencias experimentales mediante el trabajo autónomo conducente hacia una mayor comprensión del conocimiento y conceptos científicos, concebido como premisa para que los docentes en su desempeño profesional puedan transmitirlos al alumnado de educación infantil. A su vez, constituye un intento por salir de la reproducción en el aula y fomentar el aprendizaje desde el interés por la ciencia del propio alumnado. 
La microenseñanza (Allen y Gross, 1965; Allen, 1966; Allen y Clarck, 1967) es la metodología escogida en este estudio. Esto se debe a que esta simula las condiciones del aula donde se practican algunas habilidades y competencias para el futuro profesional en un periodo corto de tiempo. Se inició en la Universidad de Stanford y pronto tuvo una gran repercusión y amplia aceptación en un elevado número de países, independiente de las características del sistema educativo. Además de las mejoras en las habilidades docentes (Peleberg, 1970), cabe destacar las opciones dentro del proceso de enseñanza y aprendizaje para la consecución de las competencias docentes. Este autor anunció su carácter innovador y su práctica en contextos cerrados controlados, laboratorios de investigación adaptados a las necesidades de esta metodología.

Así, en España llegó en el curso de los años 1969-1970, siendo 1969 cuando se iniciaron las primeras experiencias desde la Secretaría General Técnica del Ministerio de Educación (Ortuño, 1972; Casado, 2015); aunque existe cierta disputa por el primer Instituto de Ciencias de la Educación que inició la metodología para la preparación de los futuros docentes. Ortuño (1972) sitúa este inicio en el ICE de la Universidad Autónoma de Barcelona, mientras que Casado (2015) lo hace en el ICE de la Universidad de Santiago de Compostela.

Para la metodología de la microenseñanza, existe un método, así como recomendaciones de empleo. Se suele encomendar no más de 15 minutos, donde se practica un concepto atendiendo a las competencias docentes de forma muy específica y concreta. De esta manera, se desgrana el proceso de enseñanza y aprendizaje controladamente en componentes específicos: de la transmisión de conocimientos y de la actuación como profesional de la educación con lo que esta última implica sean saberes, actitudes y procederes en el aula. Esta metodología presenta dos características: las sesiones son grabadas y la retroalimentación que permiten estas grabaciones. Estas dos características consiguen que el alumno reflexione acerca de su acción docente, al posicionarlo delante de un grupo reducido y realizar un esfuerzo en la transmisión de conceptos complejos; sin olvidar el guía a sus compañeros durante la realización de los experimentos propuestos (Sabelli, Ornique y Giovannini, 2014).

Al presentar el problema partimos desde el interés personal del alumnado por los temas relacionados con las ciencias de la naturaleza y por sus conocimientos iniciales que contribuyen a la construcción de nuevos conceptos, asentando mejor aquellos que inicialmente presentaban (Ausubel et al., 1983). Dichos conocimientos deben considerarse el aprender a conocer, el aprender a hacer, el aprender a convivir y el aprender a ser (Delors, 1996); todos ellos válidos para el desarrollo de las personas a lo largo de la vida. Este desarrollo se encuentra recogido en la Nueva Agenda de las Capacidades para Europa (Bachmann y Holdsworth, 2016), en la que se defiende la adquisición de un amplio abanico de competencias claves, las cuales son las que orientan hacia el aprendizaje a lo largo de la vida, y las que dan paso a una serie de competencias específicas, según el campo profesional en el que se desarrollan las personas.

En el ámbito de la educación, y durante la formación inicial de las futuras maestras, las competencias específicas que se pretenden conseguir en los egresados de los grados de educación infantil durante sus años de formación se recogen en la Orden ECI/3854/2007 y se perciben los saberes de Delors (1996). La mayoría de las investigaciones centradas en la microenseñanza se orientan a la formación inicial de futuros maestros y profesores en algún momento de sus etapas formativas (Villar, 1982; Cooper, 1989; Watson, 2007). Esta formación inicial del profesorado se encamina hacia la búsqueda de una formación global del alumnado, en la cual los conocimientos son un elemento más de esta formación, pero no el único. Son formaciones desde las que la práctica en las aulas se considera parte de esta etapa educativa, y mediante las cuales se integran los saberes de Delors (1996). 
En este sentido, el estudio de Alonso, Martini y Ormaechea (2003) adquiere relevancia al presentar la metodología de la microenseñanza como un punto de unión entre la teoría dada en el aula y las prácticas externas que se realizan durante los años de formación inicial desde las facultades de Ciencias de la Educación en instituciones externas con las que se tienden lazos de colaboración. Estos autores encuentran en la microenseñanza el elemento que en las aulas contribuyen a superar los primeros obstáculos que el alumnado se encontraría en los centros de prácticum. Contribuyendo a la aportación de estos autores, se incide en la propuesta de ChevaIlard (1991): la transposición didáctica. En este concepto "el paso del saber sabio al saber enseñado" (p. 15), es lo que se persigue en las prácticas de laboratorio y cómo, además de los conceptos científicos, el alumnado debe ser capaz de adaptar el lenguaje para ser entendido durante la explicación a la vez que debe ser capaz de mostrar aspectos propios de su futura función docente. Todo esto debe conseguirse sin perder el vocabulario científico.

A este concepto teórico se debe añadir la propuesta de Chi y Roscoe (2002), al pedir que las compañeras anticipen los resultados apareciendo las ideas previas que estas tienen. Estos autores formulan la teoría en la cual las ideas previas son mecanismos cognitivos mediante los cuales consiguen interpretar, entender y explicar la realidad; en cierta manera, un modo de acercarse a ella. Estos autores proponen una metodología que permite superar y ajustar la explicación inicial, consiguiendo definir la realidad, porque las explicaciones que se dan se acercan cada vez más a ella.

Además de las disciplinas orientadas a la formación de futuros docentes, cabe considerar aquel uso de la metodología de la microenseñanza para la mejora de la función docente, para que los profesores y maestros en activo puedan perfeccionar su quehacer en el aula (Guillermo, 1997). Con esto se incide en la capacitación, la mejora de habilidades docentes y en la contribución a la adquisición de distintas técnicas desde la reflexión y la retroalimentación que permite esta metodología.
La reflexión es el elemento que el autor determina como relevante en la formación de habilidades del profesorado en activo, ya que la cotidianidad de la función docente no prioriza la reflexión personal sobre la práctica realizada en el aula.

Otro campo pertinente en el uso de este tipo de metodología para la formación de futuros profesionales se da en las Ciencias de Actividades Físicas. En este ámbito, se encuentran un primer agrupamiento de investigaciones orientadas a la adquisición de competencias para los futuros profesionales (Fernández, 2015); y, un segundo grupo, aquellas que realizan una revisión de las distintas metodologías de aula que maestros y profesores de las distintas etapas educativas pueden aprovechar para la mejora del aprendizaje de su alumnado (Carrera, 2015; Zapatero, 2017). En este último caso, la contrastación y la comparación que realizan los autores de los distintos tipos de metodologías tratan de los beneficios que estas aportan al aprendizaje y cómo la microenseñanza desde la práctica y desde la vivencia de las actividades realizadas contribuyen al aprendizaje de aspectos concretos y específicos de los planes curriculares.

Dentro del ámbito universitario también se utiliza la microenseñanza para la formación de los futuros profesionales. Algunos ejemplos son: Logopedia y Psicología (Hernández-Jorge y de la Rosa, 2018) para las mejoras comunicativas; las Ciencias Biológicas (Pastorino, Correa y Raffaini, 2016; Torres, 2017) o la formación de futuros médicos (Motta-Ramírez y Hernández-Molina, 2016). En todas ellas, tratan de simular situaciones del futuro profesional para acercar al alumnado a aquello que se podrán encontrar en escenarios reales. En el caso de las Ciencias Biológicas, se trata de la formación de los futuros profesores de Biología para secundaria y cómo mejorar las capacidades propias de esa dedicación. Estas mejoras de las capacidades propias de la profesión son las que estudian los autores desde el campo de la medicina y cómo la microenseñanza constituye la metodología mediante la cual se consigue mejorar en esas capacidades y habilidades para los futuros cirujanos del 
ejército desde situaciones simuladas, y facilitando la reflexión posterior.

Desde la enseñanza de las Ciencias de la $\mathrm{Na}$ turaleza para las futuras maestras de educación infantil, esta metodología de la microenseñanza permite contribuir a su alfabetización científica para ser capaces de transmitir la cultura científica a sus futuros alumnos. Esta también los acerca a la perspectiva ciencia-tecnología-sociedad-ambiente, cada vez más en boga en el mundo de la enseñanza de las Ciencias de la Naturaleza y Experimentales (García Ruiz y Castro, 2005; Fernandes, Pires y Villamañán, 2014; Amaro et al., 2015; Fernández y Bravo, 2015; Quijano, 2016; Fernandes, Pires y Delgado-Iglesias, 2016). Esta perspectiva presenta un enfoque más global al enseñar las ciencias en la etapa de la educación infantil y se consigue: aprender ciencias, aprender acerca de las ciencias y hacer ciencia. Se busca la relación existente entre el concepto científico que hay detrás de cada experimento propuesto por el alumnado y su vinculación con la tecnología; así como la repercusión en la sociedad, donde se da ese concepto científico y cómo contribuye en nuestro día a día, especialmente en el medioambiente.

Esta microdocencia está orientada a introducirse en los saberes científicos y su demostración y explicación al resto de la clase mediante experimentos. De ahí que la finalidad de esta investigación sea ahondar en el uso de la microenseñanza como metodología adecuada para la enseñanza de los contenidos de las Ciencias de la Naturaleza. Esta se orienta hacia la formulación de los siguientes objetivos: primero, analizar la metodología de la microenseñanza como facilitador en la adquisición de saberes relacionados con la enseñanza de las Ciencias de la Naturaleza; y, segundo, correlacionar variables mediante las cuales poder orientar futuros estudios en la misma dirección.

\section{Metodología}

Para resolver los objetivos planteados, la investigación se sitúabajo una perspectiva empírico-analítica de corte cuantitativo. Con una metodología no experimental con un diseño de grupo único no equivalente con tratamiento de medidas solo posprueba; siendo los métodos usados el descriptivo y el correlacional tanto para describir el cambio de metodología como para acercarse a una mejor explicación de las relaciones entre las distintas variables (Latorre et al., 2005). Este tipo de metodología de investigación en educación presenta el inconveniente de tener un bajo grado de validez en los resultados. Por el contrario, permite conseguir datos pertinentes que orienten a futuros estudios, así como averiguar acerca de la viabilidad en la implantación de la metodología de la microenseñanza en el laboratorio de ciencias.

La muestra es toda la población de alumnas del grupo A de segundo del grado educación infantil en la Universidade da Coruña. El total de individuos de la población es de 65 alumnas, de las cuales conforman la muestra productora de datos 61. La mortandad es debida a la aplicación de la posprueba únicamente a aquellas alumnas que participan en las prácticas. Así, se excluyó a dos alumnas que optaron por la modalidad no presencial y a dos alumnas que no asistieron a ninguna de las sesiones durante el curso. Es, por lo tanto, un grupo constituido y natural, de elección no al azar, en el que no se pretende modificar sino observar aquello acaecido en el pasado.

Se ha construido un instrumento ad-hoc para el estudio realizado. Es un cuestionario mixto con preguntas de respuestas abiertas de poca extensión, y respuestas cerradas siguiendo una escala estimativa gráfica con distintos grados según el ítem. Son un total de 13 ítems que responden toda la muestra. Este cuestionario está separado en tres partes. Una primera compuesta de cinco ítems referentes a cuestiones psicosociales; una segunda parte, relativa a los aprendizajes con cuatro ítems; y, los últimos cuatro ítems, para la última parte que da respuesta a las consideraciones del alumnado relativas a las sesiones prácticas en el laboratorio, así como del lugar donde se realizó la experiencia 
con la metodología de la microenseñanza. Se mide la fiabilidad del instrumento con el coeficiente de la alfa de Cronbach. Se obtiene un resultado de 0.836 suficiente para considerar aceptable y poder tomar decisiones (Rincón et al., 1995).

El análisis de los datos cuantitativos obtenidos con este instrumento se realizó usando el programa SPSS-22. El proceso seguido para el análisis se inició en las pruebas de frecuencia y de descripción para cada una de las variables, continuó con la correlación de Pearson por pares de ítems, y terminó con el contraste de hipótesis para aquellos pares de variables con correlación significativa. Para los datos cualitativos, se usó el programa N-Vivo 10. En este caso, se ha utilizado la técnica de la relación para las respuestas cualitativas con la conformación de clústeres y agrupación de respuestas según similitud de estas con el coeficiente de Pearson.

\section{Resultados}

\section{Aspectos psicosociales}

En este campo dentro del cuestionario se pregunta al alumnado acerca de su titulación previa, la edad, si son repetidoras de la materia, el grupo interactivo al cual pertenecen y qué dedicación a los estudios tienen.

La edad del alumnado se encuentra en el intervalo de 19 a 30 años, siendo la media 21,62 y una moda 19. A partir de la prueba de Kolmogorov-Smirnov para una muestra, rechazamos la hipótesis nula de la distribución normal de esta al mostrar una significación asintótica con un nivel de significancia del 0,05. De esta manera, se usa la prueba no paramétrica de Wilcoxon conservando la hipótesis nula de la mediana de la edad igual a 21 años.

Para la titulación previa, el 50,8 \% del alumnado proviene de bachillerato mientras que un $41 \%$ son alumnas de ciclo formativo de educación infantil. El resto del porcentaje corresponde a: $3,3 \%$ a otros ciclos formativos y $4,9 \%$ a otros estudios universitarios. Los grupos interactivos son tres con la siguiente relación de porcentajes: 39,3 \% para el interactivo 1; 24,6 \% para el interactivo $2 ;$ y $36,1 \%$ para el interactivo 3 . Para la dedicación a los estudios se ha agrupado en aquellos que únicamente estudian, de aquellos que tienen algún tipo de trabajo. En todo caso, ninguna de las alumnas tiene un trabajo a tiempo completo. Los porcentajes para estos dos grupos son $73,8 \%$ de dedicación exclusiva a los estudios y $26,2 \%$ del alumnado con algún tipo de trabajo. Una alumna repite la materia del total de 61 que conforma la muestra.

En el contraste de hipótesis se usa la prueba binomial para una muestra para las variables "dedicación a los estudios" y "repetición de la materia". En el resto de las variables, utilizamos la prueba Chi-cuadrado. Se asume la hipótesis nula de igualdad de probabilidades en las respuestas dadas para el ítem de grupos interactivos. Para el resto, no se asume la igualdad de probabilidades entre grupos.

\section{Aprendizaje}

En este apartado se pregunta acerca del grado de acercamiento a los conceptos científicos como grupo tutor (ítem 6), al grado de acercamiento a los conceptos científicos como grupo participante (ítem 7), grado de aceptación de las ciencias antes de empezar el curso (ítem 8), y cómo ha mejorado el grado de aceptación a las ciencias al final de las prácticas (ítem 9). Véanse los porcentajes en la tabla 1 para los tres primeros ítems. Las repuestas presentan los siguientes grados: total, mucho, bastante, poco y nada; excepto el último ítem, cómo ha mejorado el grado de aceptación a las ciencias al final de las prácticas, que sigue los siguientes grados: ha mejorado, sigue igual y ha empeorado.

En los ítems de acercamiento a los conceptos científicos, únicamente se dan respuestas en los grados medio-alto, consiguiendo mayor adquisición de los conceptos cuando se está como grupo 
participante. El acercamiento a los conceptos científicos como grupo tutor (encargado de preparar y exponer los experimentos al resto de la clase) presenta igualdad de probabilidades entre los grupos; mientras que, para el grupo participante, se rechaza que esos conocimientos se producen con igualdad de probabilidades. En el grado de aceptación de las ciencias de la naturaleza al inicio del curso, las alumnas presentan tres tipos de respuestas, destacando un 14,8 \% que su aceptación es total y no existiendo nadie que exprese nada de acercamiento, aunque casi un tercio se sitúan ante poco acercamiento, poco más de un tercio consideran que es bastante y el resto de las respuestas consideran que mucho. A estas respuestas, el grado de aceptación a las Ciencias de la Naturaleza al final del curso o bien aumenta con $72,1 \%$ o bien se mantiene con $27,9 \%$; ninguna de las alumnas expresa que empeoró. En este último caso, el contraste de hipótesis entre estas dos últimas variables nos indica que no existe ni distribución ni igualdad de medianas entre los grados de aceptación de las Ciencias de la Naturaleza al inicio del curso con las del final. Lo que evidencia un aumento en el grado de aceptación general del alumnado.

Tabla 1. Porcentajes ítems segundo bloque.

\begin{tabular}{cccccc}
\hline & Nada & Poco & Bastante & Mucho & Total \\
Ítem 6 & 0 & 0 & $63,9 \%$ & $36,1 \%$ & 0 \\
Ítem 7 & 0 & 0 & $72,1 \%$ & $27,9 \%$ & 0 \\
Ítem 8 & 0 & $32,8 \%$ & $34,4 \%$ & $18,0 \%$ & $14,8 \%$ \\
\hline
\end{tabular}

Fuente: elaboración propia a partir de datos obtenidos desde el SPSS-22.

En el contraste de hipótesis, se producen las distintas respuestas en igualdad de probabilidades. Al respecto, se emplea la prueba binomial para todas las variables de este apartado y se rechaza la hipótesis nula de existencia de igualdad de probabilidades entre respuestas, se usó la prueba
Chi-cuadrado para una muestra, y en este caso, se conserva la hipótesis nula.

\section{Correlaciones}

Las variables cuantitativas presentadas anteriormente se agrupan por pares para realizar la correlación de Pearson. En la tabla 2, presentamos la relación de pares en la cual podemos comprobar la existencia de seis pares en los que existe correlación significativa: (1) grado de aceptación de las ciencias al inicio y el grado de aceptación de las ciencias al final; (2) grado de aceptación de las ciencias al inicio y el grado de conceptos científicos como grupo tutor; (3) titulación previa y edad; (4) dedicación a los estudios y edad; (5) grado concepto científico grupo tutor y grado concepto científico grupo participante; y (6) años y repetición de la materia. Se puede observar que estos pares presentan un nivel de significación inferior a 0,01 excepto el par dedicación a los estudios y edad que la significación es inferior a 0,05. Para estas seis correlaciones cabe destacar que los pares (1) y (6) son correlaciones inversas en las que para el aumento de la primera disminuye la segunda mientras que para el resto de pares la correlación es directamente proporcional: al aumentar la primera lo hace igual la segunda. De esta manera, cuando el grado de aceptación de las ciencias al inicio del curso es alto, al final baja; y cuando mayores son las alumnas menos repiten la materia.

A esto seis pares correlacionados, les aplicamos la prueba T de muestras emparejadas. Únicamente, en el par grado concepto científico grupo tutor y grado concepto científico grupo participante se acepta la hipótesis nula tanto de distribuciones iguales de grupos para la prueba de Kendall como para la mediana de diferencias cero para la prueba de Wilcoxon. El resto de pares rechazamos la hipótesis nula y se acepta la alternativa de distribuciones no iguales, según la prueba de Kendall y la mediana de diferencias no es cero para la prueba de Wilcoxon, como se muestra en la tabla 3. 
Tabla 2. Resultados de las correlaciones de Pearson.

\begin{tabular}{|c|c|c|c|c|c|c|c|c|c|c|}
\hline \multicolumn{11}{|c|}{ Correlaciones } \\
\hline & & $\begin{array}{l}\text { grado_acep_- } \\
\text { CCNN_inicio }\end{array}$ & $\begin{array}{l}\text { grado_acep_- } \\
\text { CCNN_final }\end{array}$ & Titu_prev & $\begin{array}{c}\text { grupo_interac } \\
\text { tivo }\end{array}$ & $\begin{array}{c}\text { dedica_estud } \\
\text { ios } 1-2\end{array}$ & $\begin{array}{c}\text { grado_conce } \\
\text { pt_cient_GTut } \\
\text { or }\end{array}$ & $\begin{array}{c}\text { grado_conce } \\
\text { pt_cient_Gpar } \\
\text { tic }\end{array}$ & Edad_Años & repe_materia \\
\hline \multirow{5}{*}{$\begin{array}{l}\text { grado_acep_CCNN_inici } \\
0\end{array}$} & Correlación de Pearson & 1 &,$- 475^{\mathrm{Nx}}$ &,- 131 & .140 & 013 & $.354^{\mathrm{Nx}}$ & .053 &,- 009 &,- 018 \\
\hline & Sig. (bilateral) & &, 000 &, 315 &, 281 & 921 &, 005 & 687 & 946 & 888 \\
\hline & $\begin{array}{l}\text { Suma de cuadrados y } \\
\text { productos vectoriales }\end{array}$ & 65,672 & $-13,492$ & $-6,393$ & 7,705 &, 361 & 10,754 & 1,492 & $-1,393$ &,- 148 \\
\hline & Covarianza & 1,095 &,- 225 &,- 107 &, 128 &, 006 &, 179 &, 025 &,- 023 &,- 002 \\
\hline & $\mathrm{N}$ & 61 & 61 & 61 & 61 & 61 & 61 & 61 & 61 & 61 \\
\hline \multirow[t]{5}{*}{ grado_acep_CCNN_final } & Correlación de Pearson &,$- 475^{\mathrm{NI}}$ & 1 &,- 028 &,- 145 & $\begin{array}{l}-038 \\
\end{array}$ & 086 & 142 &,- 125 & 080 \\
\hline & Sig. (bilateral) &, 000 & &, 831 &, 265 &, 770 &, 509 &, 276 &, 335 &, 539 \\
\hline & $\begin{array}{l}\text { Suma de cuadrados y } \\
\text { productos vectoriales }\end{array}$ & $-13,492$ & 12,262 &,- 590 & $-3,443$ &,- 459 & 1,131 & 1,738 & $-8,590$ & .279 \\
\hline & Covarianza &,- 225 &, 204 &,- 010 &,- 057 &,- 008 & .019 & .029 &,- 143 &, 005 \\
\hline & $\mathrm{N}$ & 61 & 61 & 61 & 61 & 61 & 61 & 61 & 61 & 61 \\
\hline \multirow[t]{5}{*}{ Titu_prev } & Correlación de Pearson &,- 131 &,- 028 & 1 & , 104 & , 195 &, 120 & 028 & $.529^{112}$ &,- 063 \\
\hline & Sig. (bilateral) &, 315 &, 831 & &, 425 &, 133 &, 358 & 831 &, 000 & 629 \\
\hline & $\begin{array}{l}\text { Suma de cuadrados y } \\
\text { productos vectoriales }\end{array}$ & $-6,393$ &,- 590 & 36,328 & 4,246 & 4,033 & 2,705 &, 590 & 62,328 &,- 377 \\
\hline & Covarianza &,- 107 &,- 010 &, 605 & 071 &, 067 &, 045 &, 010 & 1,039 &,- 006 \\
\hline & $\mathrm{N}$ & 61 & 61 & 61 & 61 & 61 & 61 & 61 & 61 & 61 \\
\hline \multirow[t]{5}{*}{ grupo_interactivo } & Correlación de Pearson & 140 &,- 145 & , 104 & 1 & 151 & .090 & 103 &,- 006 &,- 154 \\
\hline & Sig. (bilateral) & 281 &, 265 &, 425 & &, 244 & .492 &, 430 & 965 &, 237 \\
\hline & $\begin{array}{l}\text { Suma de cuadrados y } \\
\text { productos vectoriales }\end{array}$ & 7,705 & $-3,443$ & 4,246 & 45,934 & 3,525 & 2,279 & 2,443 &,- 754 & $-1,033$ \\
\hline & Covarianza & 128 &,- 057 & 071 &, 766 &, 059 & .038 &, 041 &,- 013 &,- 017 \\
\hline & $\mathrm{N}$ & 61 & 61 & 61 & 61 & 61 & 61 & 61 & 61 & 61 \\
\hline \multirow[t]{5}{*}{ dedica_estudios 1-2 } & Correlación de Pearson &, 013 &,- 038 & , 195 & 151 & 1 &,- 095 &,- 045 & $254^{x}$ & 077 \\
\hline & Sig. (bilateral) & 921 &, 770 &, 133 &, 244 & &, 464 &, 731 & .049 &, 555 \\
\hline & $\begin{array}{l}\text { Suma de cuadrados y } \\
\text { productos vectoriales }\end{array}$ &, 361 &,- 459 & 4,033 & 3,525 & 11,803 & $-1,230$ &,- 541 & 17,033 &, 262 \\
\hline & Covarianza &, 006 &,- 008 &, 067 &, 059 &, 197 &,- 020 &,- 009 &, 284 &, 004 \\
\hline & $\mathrm{N}$ & 61 & 61 & 61 & 61 & 61 & 61 & 61 & 61 & 61 \\
\hline \multirow{5}{*}{$\begin{array}{l}\text { grado_concept_cient_GT } \\
\text { utor }\end{array}$} & Correlación de Pearson & $354^{\prime \prime \prime}$ & 086 & .120 & .090 &,- 095 & 1 & $371^{m}$ & 228 &,- 097 \\
\hline & Sig. (bilateral) &, 005 &, 509 &, 358 &, 492 & .464 & &, 003 & .077 & .457 \\
\hline & $\begin{array}{l}\text { Suma de cuadrados y } \\
\text { productos vectoriales }\end{array}$ & 10,754 & 1,131 & 2,705 & 2,279 & $-1,230$ & 14,066 & 4,869 & 16,705 &,- 361 \\
\hline & Covarianza &, 179 & .019 &, 045 &, 038 &,- 020 &, 234 & 081 &, 278 &,- 006 \\
\hline & $\mathrm{N}$ & 61 & 61 & 61 & 61 & 61 & 61 & 61 & 61 & 61 \\
\hline \multirow{5}{*}{$\begin{array}{l}\text { grado_concept_cient_Gp } \\
\text { artic }\end{array}$} & Correlación de Pearson & .053 & .142 & 028 & 103 &,- 045 & $.371^{\pi x}$ & 1 & .096 & 208 \\
\hline & Sig. (bilateral) &, 687 &, 276 & 831 &, 430 &, 731 &, 003 & & .461 & , 108 \\
\hline & $\begin{array}{l}\text { Suma de cuadrados y } \\
\text { productos vectoriales }\end{array}$ & 1,492 & 1,738 &, 590 & 2,443 &,- 541 & 4,869 & 12,262 & 6,590 &, 721 \\
\hline & Covarianza & .025 &, 029 & .010 & 041 &,- 009 & 081 & 204 &, 110 &, 012 \\
\hline & $\mathrm{N}$ & 61 & 61 & 61 & 61 & 61 & 61 & 61 & 61 & 61 \\
\hline \multirow[t]{5}{*}{ Edad_Años } & Correlación de Pearson &,- 009 &,- 125 & $.529^{\prime \prime}$ &,- 006 & $254^{x}$ & 228 & 096 & 1 &,$- 329^{n \pi}$ \\
\hline & Sig. (bilateral) &, 946 &, 335 & .000 & 965 & .049 &, 077 & .461 & &, 010 \\
\hline & $\begin{array}{l}\text { Suma de cuadrados y } \\
\text { productos vectoriales }\end{array}$ & $-1,393$ & $-8,590$ & 62,328 &,- 754 & 17,033 & 16,705 & 6,590 & 382,328 & $-6,377$ \\
\hline & Covarianza &,- 023 &,- 143 & 1,039 &,- 013 &, 284 &, 278 &, 110 & 6,372 &,- 106 \\
\hline & $\mathrm{N}$ & 61 & 61 & 61 & 61 & 61 & 61 & 61 & 61 & 61 \\
\hline \multirow[t]{5}{*}{ repe_materia } & Correlación de Pearson &,- 018 & .080 &,- 063 & $\begin{array}{l}, 154 \\
\end{array}$ & 077 &,- 097 & 208 &,$- 329^{\prime \prime}$ & 1 \\
\hline & Sig. (bilateral) &, 888 &, 539 &, 629 &, 237 &, 555 &, 457 & , 108 &, 010 & \\
\hline & $\begin{array}{l}\text { Suma de cuadrados y } \\
\text { productos vectoriales }\end{array}$ &,- 148 & 279 &,- 377 & $-1,033$ & .262 &,- 361 &, 721 & $-6,377$ & 984 \\
\hline & Covarianza &,- 002 &, 005 &,- 006 &,- 017 & .004 &,- 006 & .012 &,- 106 &, 016 \\
\hline & $\mathrm{N}$ & 61 & 61 & 61 & 61 & 61 & 61 & 61 & 61 & 61 \\
\hline
\end{tabular}

**. La correlación es significativa en el nivel 0,01 ( 2 colas)

*. La correlación es significativa en el nivel 0,05 ( 2 colas)

Fuente: elaboración propia. 
Tabla 3. Resultados contraste de hipótesis para muestras emparejadas.

\begin{tabular}{|c|c|c|c|c|c|c|c|c|c|}
\hline \multicolumn{10}{|c|}{ Prueba de muestras emparejadas } \\
\hline & & \multicolumn{5}{|c|}{ Diferencias emparejadas } & \multirow[b]{3}{*}{ t } & \multirow[b]{3}{*}{$\mathrm{gl}$} & \multirow[b]{3}{*}{ Sig. (bilateral) } \\
\hline & & \multirow[b]{2}{*}{ Media } & \multirow{2}{*}{$\begin{array}{l}\text { Desviación } \\
\text { estándar }\end{array}$} & \multirow{2}{*}{$\begin{array}{l}\text { Media de } \\
\text { error } \\
\text { estándar }\end{array}$} & \multicolumn{2}{|c|}{$\begin{array}{l}\text { 95\% de intervalo de confianza } \\
\text { de la diferencia }\end{array}$} & & & \\
\hline & & & & & Inferior & Superior & & & \\
\hline Par 1 & $\begin{array}{l}\text { grado_acep_CCNN_inici } \\
0- \\
\text { grado_acep_CCNN_final }\end{array}$ & 1,574 & 1,322 & 169 & 1,235 & 1,912 & 9,295 & 60 & ,000 \\
\hline Par 2 & $\begin{array}{l}\text { grado_acep_CCNN_inici } \\
0- \\
\text { grado_concept_cient_GT } \\
\text { utor }\end{array}$ & 1,213 &, 985 &, 126 & ,961 & 1,465 & 9,618 & 60 &, 000 \\
\hline Par 3 & Titu_prev - Edad_Años & $-20,000$ & 2,214 & ,283 & $-20,567$ & $-19,433$ & $-70,566$ & 60 &, 000 \\
\hline Par 4 & $\begin{array}{l}\text { dedica_estudios 1-2 - } \\
\text { Edad_Años }\end{array}$ & $-20,361$ & 2,450 &, 314 & $-20,988$ & $-19,733$ & $-64,914$ & 60 &, 000 \\
\hline Par 5 & $\begin{array}{l}\text { grado_concept_cient_GT } \\
\text { utor- } \\
\text { grado_concept_cient_Gp } \\
\text { artic }\end{array}$ &,- 082 &, 526 &, 067 &,- 217 &, 053 & $-1,217$ & 60 &, 228 \\
\hline Par 6 & $\begin{array}{l}\text { Edad_Años - } \\
\text { repe_materia }\end{array}$ & 19,639 & 2,569 &, 329 & 18,981 & 20,297 & 59,701 & 60 &, 000 \\
\hline
\end{tabular}

Fuente: elaboración propia a partir de los datos analizados desde el SPSS-22.

\section{Sesiones prácticas}

En este apartado los ítems son de respuesta corta; excepto la que pregunta acerca del interés por la metodología usada en las sesiones prácticas, en las cuales el $100 \%$ del alumnado contestan afirmativamente el interés por esta metodología. Se usa la prueba binomial para una muestra para realizar el contraste de hipótesis para esta variable. Se rechaza la hipótesis nula de igualdad de probabilidades.

Respecto a las dificultades con las que se encontraron las alumnas para realizar las prácticas, y después de agrupar en clústeres las respuestas dadas, cabe destacar que cerca del $13 \%$ de las respuestas consideran que no existieron dificultadas. El resto de respuestas se pueden agrupar en la organización de las sesiones con un $35 \%$, seguida por las dificultades de presentar los experimentos ante el resto de compañeras con más del $16 \%$. Otra de las dificultadas está en el uso del vocabulario científico con más de un $9 \%$ de las respuestas. Entre las menos contestadas, se encuentran: el material del laboratorio, $7 \%$; el espacio limitado del laboratorio, $5 \%$; el horario de tarde, 2,5\%; y, la poca utilidad en la vida cotidiana, 1,3\%.
También se preguntó por las ventajas de esta metodología. Se pueden agrupar las respuestas en los siguientes clústeres: adquisición de conocimientos, 30 \%; las distintas fases de elaboración de las prácticas como el trabajo en grupo, la exploración, la manipulación y la experimentación con un $30 \%$; un $18 \%$ hacen referencia a la metodología por tres motivos, primero, el interés que suscita, segundo, el dinamismo que se da a las sesiones $y$, tercero, por considerar innovadora la metodología; el carácter lúdico de las sesiones, $15 \%$. Cierran las respuestas: la guía del profesor, 4 \%; y, su contribución a la mejora para exponer ante el grupo, $3 \%$.

Por último, se preguntó por tres mejoras a las prácticas llevadas a cabo. No todo el alumnado respondió a la cantidad propuesta y lo hacen con menos. Las respuestas se agrupan en tres grandes bloques: 29,91 \% como mejoras en la organización institucional; 49,53\% mejoras en la propia organización de las prácticas; y, 18,69 \% de mejoras relacionadas con el propio alumnado. Destacan dos alumnas que responden que no mejorarían nada justificándolo una de ellas al decir que la metodología es participativa y facilita el aprendizaje 
significativo de los conceptos científicos tratados durante las prácticas. Dentro de las mejoras institucionales se pide más material y un espacio más amplio. También se pide eliminar el horario de tarde y el aumento de horas de prácticas. En las mejoras propias de la organización de las prácticas, destacan las aportaciones relacionadas con salidas bien sean de campo o salidas a centros escolares para presentar experimentos científicos y llevarlos a cabo con el alumnado de la etapa de infantil. Otras mejoras que indica el alumnado son: aumentar el tiempo tanto para la comprobación correcta de los experimentos que se realizarán como de la realización de cada experimento. Hay algunas respuestas que prefieren aumentar el número de experimentos por grupo, aunque algunas alumnas prefieren disminuirlos.

Por último, dentro de este gran bloque de respuestas, hay alumnas que piden la no utilización de comida porque esta termina tirándose después de los experimentos. En el último bloque de mejoras propuestas por las alumnas relacionadas con ellas, la mayoritaria es la aportación que indican mejoras en las explicaciones dadas por el grupo tutor, seguida por la mayor atención por parte del resto de compañeras. A esto cabe añadir respuestas que piden unas explicaciones más simples y mejorar la comprobación en la comprensión de los conceptos explicados.

\section{Discusión}

Se empieza esta discusión dando respuesta a la consecución de los objetivos de esta investigación. Para el primero de ellos, analizar la metodología de la microenseñanza como facilitador en la adquisición de saberes relacionados con la enseñanza de las ciencias de la naturaleza, se debe recordar que una de las bases del estudio está en los pilares de Delors (1994): aprender a conocer, aprender a hacer, aprender a convivir y aprender a ser. Cierto que el instrumento se orienta al primer pilar, pero no se puede obviar que en las respuestas del último bloque se aprecian indicaciones acerca de los otros tres pilares. Para el primer pilar se considera que la metodología es adecuada porque en los resultados obtenidos hay un aumento en la adquisición de los conceptos científicos y también en la aceptación de las ciencias de la naturaleza, en la que se observa un aumento significativo del inicio del curso al final. También, se puede considerar la adquisición de conocimientos expresada en la pregunta de ventajas de esta metodología.

En este aprendizaje se hace necesario, para futuras investigaciones, concretar la adquisición de los conceptos científicos, así como comprobar cuántos de los alumnos se matriculan en los siguientes cursos en las materias optativas relacionadas con las ciencias de la naturaleza (salud y medioambiente) ofrecidas en tercero y cuarto grado. Para los otros tres pilares, no existen ítems cuantitativos que se acercan a ellos, pero en los ítems finales se pregunta acerca de las dificultades encontradas y las mejoras que propondrían. En las dificultades, el uso del vocabulario científico y el material disponible para algunos de los experimentos estarían en línea con el aprender a hacer, a ser reflexivas respecto a su futura calificación profesional y a ser capaces de hacer frente a distintas situaciones, en este caso los experimentos en el laboratorio y distintas soluciones. Aunque en el caso del vocabulario fue solo el $9 \%$ del alumnado quien expresó esas dificultas que nos indica ese grado de aceptación a las ciencias que existiendo casi un tercio al inicio del curso que era poca, al final del curso o bien habían mejorado o estaba igual que al principio (Campanario y Moya, 1999).

El material también formaría parte del pilar, aprender a ser, por la autonomía presentada en la preparación y realización de los experimentos porque les permitió ajustarse a lo existente. Este pilar también se puede ver reflejado en las reflexiones realizadas por las alumnas en las mejoras que propondrían como las propias de la organización institucional y las relativas a la organización de las prácticas, especialmente en el espacio reducido para realizar las prácticas o incluso el poder 
Ilevarlas a cabo en otros espacios más abiertos fuera del propio laboratorio.

Por último, el pilar aprender a convivir se expresan en las dificultadas de presentar los experimentos al resto de compañeras $y$, en algunos casos, el resto de grupos demuestran poco respeto al grupo tutor, permite esa autoevaluación (Özcan y Gerçek, 2019) y coevaluación desde la reflexión de todo el acto educativo; además de cierto estrés en el grupo tutor (Horgan, Howard y Gardiner-Hyland, 2018) que sería posiblemente lo que causa ese bajo valor en el grado de aceptación final respecto al presentado al inicio. Esto contrasta con la importancia que otorga el alumnado al acto de experimentar y las vivencias en el laboratorio (Cantó, Pro y Solbes, 2016). Se formula en palabras que indican la poca comprensión por el otro, por el poco respeto por parte del grupo pasivo hacia quien lleva la sesión. En todo caso, sabiendo la existencia mostrada indirectamente por el alumnado acerca de estos tres pilares y que no fueron considerados directamente en el instrumento, se debería incluir esos aspectos en futuros estudios, así como el uso de instrumentos de control y técnicas de observación para cotejar y triangular la información obtenida y conseguir así una mayor validez externa.

El segundo objetivo, correlacionar variables mediante las cuales poder orientar futuros estudios en la misma dirección, se puede comprobar en las tablas 2 y 3 aquellas correlaciones existentes y cómo futuras investigaciones deben incluir y ampliar los conceptos que se correlacionan. Se encuentran seis parejas de correlación que nos orientan hacia el cambio en el grado de aceptación de las ciencias, como esta metodología favorece y aumenta esta aceptación en el alumnado. Cabe destacar ese $13 \%$ que considera no encontrar dificultadas con el uso de esta metodología, aunque el $100 \%$ indican interés por la metodología usada en las prácticas de laboratorio que consigue cierto aumento en la motivación (Nieto y Santiago, 2014) por las ciencias de la naturaleza. Se puede considerar un carácter reflexivo en el alumnado cuando se piden mejoras a realizar que únicamente dos alumnas consideran que no es necesario realizar cambios y cómo este carácter reflexivo va acompañado por la construcción de la capacidad de enseñanza (Lederman y Lederman, 2019), cuando el $13 \%$ no encontraron dificultades. Destaca la correlación inversamente proporcional existente entre el grado de aceptación de las ciencias al inicio respecto al grado de aceptación al final, lo que nos indica que a mayor grado de aceptación al inicio menor es el grado de aceptación al final. Esto contradice, en cierta manera, los estudios que muestran que con la práctica y metodologías más participativas aumenta la adquisición de conocimientos y el grado de aceptación (Gaete y Camacho, 2017). En el presente estudio, la correlación es contraria en el caso del alumnado con elevado grado de aceptación inicial, aunque no para los grados de aceptación bajos porque en estas situaciones aumenta al final. Se puede concluir que es una metodología adecuada para bajos conocimientos o baja aceptación en un tema.

Para concluir, se debe considerar en futuros estudios la mejora del instrumento para poder obtener una mayor fiabilidad y validez del instrumento; enfocando esa mejora en el aprender a ser y aprender a convivir. Además de añadir algún otro instrumento que permita triangular la información obtenida desde varios puntos de vista distintos.

No se puede olvidar antes de cerrar esta investigación el concepto de alfabetización científica, el cual se busca que adquiera el alumnado de educación infantil. Para ello, debemos desde las aulas de la universidad alfabetizar científicamente al alumnado que en un futuro cercano serán las maestras de los más pequeños y las que deberán tener entre otras muchas responsabilidades enseñar ciencia, enseñar a hacer ciencia y enseñar acerca de la ciencia a sus alumnos. Esta función no la podrán realizar si desde las aulas universitarias no se sienten motivas e interesadas por este campo.

Cabría proponer una vez realizado este estudio acciones educativas mucho más activas a lo largo de todas las sesiones, ya no solo las 
prácticas como en el caso de este estudio, sino que también en las expositivas mucho más teóricas, y en las que se acerca al alumnado los conceptos propios de la enseñanza de las ciencias en educación infantil.

\section{Referencias}

Allen, D. W. (1966). Microteaching: A New Framework for In-Service Education. The High School Journal, 49, 357-362.

Allen, D. W.; Clarck, R. J. (1967). Microteaching: It's Rationale. The High School Journal, 51, 75-79.

Allen, D. W.; Gross, R. E. (1965). Microteaching a New Beginning for Beginners. The NEA Journal, 55, 25-26.

Alonso, M. C.; Martini, B. A.; Ormaechea, S. (2003). Obstáculos que afectan la relación teoría-práctica en las prácticas de formación: el caso particular de los practicantes de la carrera de ciencias de la educación de la UNNE. Revista Nordeste, 18, 101-111.

Amaro, F.; Manzanal, A. I.; Cuetos, M. J. (2015). Didáctica de las Ciencias Naturales y Educación Ambiental. Logroño: UNIR.

Ausubel, D. P.; Novak, J. D.; Hanesian, H. (1983). Psicología educativa. Un punto de vista cognoscitivo. México: Trías editorial.

Bachmann, D.; Holdsworth, P. (2016). La nueva agenda para las capacidades para Europa. Educación de Adultos y Desarrollo, 83, 18-32.

Campanario, J. M.; Moya, A. (1999). ¿Cómo enseñar ciencias? Principales tendencias y propuestas. Investigaciones didácticas, 17(2), 179-192.

Cantó, J.; Pro, A. de; Solbes, J. (2016). ¿Qué ciencias se enseñan y cómo se hace en las aulas de educación infantil? La visión de los maestros en formación inicial. Enseñanza de las Ciencias, 34(3), 25-50. http://dx.doi.org/10.5565/ rev/ensciencias. 1870

Carrera, D. (2015). Tareas integradas: características. La aportación desde el área de la educación física. Revista Digital de Educación Física, 6(35), 161-175.

Casado, J. (2015). Los primeros años del Instituto de Ciencias de la Educación de la Universidad de Santiago de Compostela. Innovación Educativa, 25, 47-54.

Chevallard, Y. (1991). La transposition didactique: du savoir savant au savoir enseigné. Grenoble: La Pensée Sauvage.

Chi, M. T. H.; Roscoe, R. D. (2002). The processes and challenges of conceptual change. En M. Limon y L. Mason (eds.), Reconsidering conceptual change: Issues in theory and practice, 3-27. Dordrecht: Springer. https://doi. org/10.1007/0-306-47637-1_1

Cooper, J. M. (1989). La microenseñanza: la precursora de la formación del profesorado basada en competencias. En J. Gimeno Sacristán y A. Pérez Gómez (ed.), La Enseñanza: su teoría y su práctica (pp. 364-371). Madrid: ediciones Akal.

Delors, J. (1996). La educación encierra un tesoro. Madrid: Santilla, Ediciones Unesco.

Fernandes, I. M.; Pires, D. M.; Villamañán, R. M. (2014). Educación científica con enfoque Ciencia-Tecnología-Sociedad-Ambiente. Construcción de un instrumento de análisis de las directrices curriculares. Formación Universitaria, 7(5), 23-32. https://doi.org/10.4067/ $\underline{\text { S0718-50062014000500004 }}$

Fernandes, I. M.; Pires, D. M.; Delgado-Iglesias, J. (2016). Las relaciones entre ciencia, tecnología, sociedad y ambiente, en los libros de texto de Educación Primaria: un estudio comparativo entre Portugal y España, antes de las últimas reformas educativas. Revista Eureka sobre Enseñanza y Divulgación de las Ciencias, 14(1), 54-68. https://doi.org/10.2526/Rev Eureka ensen divulg cienc.2017.v14.i1.05

Fernández, R.; Bravo, M. (2015). Las ciencias de la naturaleza en la Educación Infantil. Madrid: Pirámide.

Fernández, M.; Espada, M. (2015). Frecuencia de utilización y aceptación de estilos de 
enseñanza en educación física. Athlos. Revista Internacional de Ciencias Sociales, de la Actividad Física, el Juego y el Deporte, 9, 44-63. http://www.museodeljuego.org

Gaete, M.; Camacho, J. (2017). Vivencias de practicantes de pedagogías en ciencias. Educaçao e Pesquisa, 43(2), 341-356. http://dx.doi. org/10.1590/s1517-9702201609146976

García-Ruiz, A.; Castro, M. D. (2005). Aplicación del enfoque ciencia, tecnología, sociedad y ambiente ante problemas reales: vertidos incontrolados de residuos. Enseñanza de las Ciencias, número extra, 1-6.

Guillermo, M. C. (1997). Microenseñanza: ¿una técnica vigente para el desarrollo de habilidades docentes. Educación y ciencia. Nueva época, 1(2), 16, 59-68.

Hernández, J. C. M.; de la Rosa, C. M. (2018). Percepción de mejora de las habilidades comunicativas en estudiantes universitarios. Revista de la Educación Superior, 47(186), 119-135.

Horgan, K.; Howard, S.; Gardiner-Hyland, F. (2018). Pre-service teachers and stress during microteaching: an experimental investigation of the effectiveness of relaxation training with biofeedback on psicological and physiological indices of stress. Applied Psychophysiology biofeedback, 42(3), 217-225. https://doi. org/10.1007/s10484-018-9401-9

Latorre, A.; Del Rincón, D.; Arnal, J. (2005). Bases metodológicas de la investigación educativa. Barcelona: Ediciones Experiencia.

Lederman, N. G.; Lederman, J. S. (2019). Teaching and Learning of Nature of Scientific knowledge and scientific inquiry: building capacity through systematic research-based professional development. Journal of Science Teacher Education. https://doi. org/10.1080/1046560X.2019.1625572

Motta-Ramírez, G. A.; Hernández-Molina, S. I. (2016). La microenseñanza: técnica de actualidad para la capacidad y formación de docentes. Revista Sanidad Militar México, 70(2), 230-234.
Nieto, A.; Santiago, P. R. (2014). Microenseñanza una técnica para motivar el enseñar y aprender investigando. Espectros, 52, 23-31.

Orden ECI/3854/ (2007). Por la que se establecen los requisitos para la verificación de los títulos universitarios oficiales que habiliten para el ejercicio de la profesión de Maestro en Educación Infantil. BOE, 312, § 22446.

Ortuño, V. (enero-febrero de 1972). La microenseñanza. Revista de Educación, número extraordinario, 10-16.

Özcan, Ö.; Gerçek, C. (2019). Multidimensional analyzing of the microteaching applications in teacher education via videograph. European Journal of Teacher Education, 42(1), 8297. https://doi.org/10.1080/02619768.2018. $\underline{1546285}$

Pastorino, I. C.; Correa, A. L. R.; Raffaini, G. B. (2016). Las actividades experimentales en la formación inicial de profesores de biología de la U.N.R.C. Educación, Formación e Investigación, 2(3), 1-11.

Peleberg, A. (1970). Microenseñanza: un innovador procedimiento de laboratorio para mejorar la enseñanza y el entrenamiento de profesores. Unesco's Bulletin Prospects in Education, 1(3), 1-15.

Quijano, R. (2016). Enseñanza de las Ciencias de la Naturaleza en Educación Infantil. Madrid: Pirámide.

Rincón, D. del; Arnal, J.; Latorre, A.; Sans, A. (1995). Técnicas de investigación en ciencias sociales. Madrid: Dykinson.

Sabelli, M.; Ornique, M.; Giovannini M. (2014). Las prácticas simuladas: entre la construcción de verosimilitud, la observación y reflexión. I Encuentro Internacional de Educación. Espacios de investigación y divulgación. Tandil, Argentina, 29, 30 y 31 de octubre.

Torres, A. M. (2017). Un polo a Tierra en la formación inicial de maestros de ciencias. Bio-grafía. Escritos sobre la Biología y su Enseñanza. IV Congreso Nacional de Investigación en Enseñanza de la Biología 
y IX Encuentro Nacional de Experiencias en Enseñanza de la Biología y la Educción Ambiental, 9-11 de octubre, Bogotá, Colombia. https://doi.org/10.17227/bio-grafia. extra2017-7312

Villar, L. M. (1982). La microenseñanza como método de formación del profesorado. Tesis doctoral inédita. Universidad de Sevilla, Sevilla.
Watson, R. (2007). La microenseñanza en la UPC. Revista Digital de Investigación en Docencia Universitaria, 3(1), 1-20. https://doi. org/10.19083/ridu.3.24

Zapatero, J. A. (2017). Beneficios de los estilos de enseñanza y las metodologías centradas en el alumno en educación física. E-balonmano.com: Revista de Ciencias del Deporte, 13(3), 237-250. 